Article

\title{
Research on Corrosion Damage Evolution of Aluminum Alloy for Aviation
}

\author{
Zhigang Gao, Yuting He * ${ }^{\mathbb{D}}$, Sheng Zhang, Tianyu Zhang and Fei Yang \\ Aeronautics Engineering College, Air Force Engineering University, Xi'an 710038, China; \\ gaozhigang666@126.com (Z.G.); zsxinzhou@126.com (S.Z.); zz664191970@126.com (T.Z.); \\ sunshinefly2018@126.com (F.Y.) \\ * Correspondence: heyut666@126.com; Tel.: +86-182-2082-1840
}

Received: 24 July 2020; Accepted: 12 October 2020; Published: 15 October 2020

check for updates

Featured Application: According to the environment of aircraft internal structure, a laboratory-accelerated corrosion method was designed to simulate Wanning area in Hainan Province of China. A simple and accurate extraction method of corrosion rate by image binarization was proposed.

\begin{abstract}
Based on the real annual average value of atmospheric environmental data in the Wanning area of Hainan Province in China by selecting 7075 ultra-high-strength aluminum alloy specimens for aviation, a new corrosion solution was designed and the traditional alternate immersion corrosion method of using the alternate immersion corrosion test box was improved to simulate the environment of the internal structure of the aircraft. On this basis, two kinds of corrosion damage parameters, the depth of corrosion pits and corrosion rate, were quickly and accurately obtained by the threedimensional profile of the specimen and binarization images' method. The optimal linear regression equation combination of pitting depth and corrosion rate was established, and the dynamic evolution equation of the depth of corrosion pits and corrosion rate was obtained. The results showed that: The depth of corrosion pits in the early stage of corrosion ( $8 \mathrm{~h}$ and $24 \mathrm{~h}$ ) obeyed the Gumbel distribution and Weibull distribution, respectively, and the later stage ( $48 \mathrm{~h}, 72 \mathrm{~h}, 96 \mathrm{~h}$, and $120 \mathrm{~h})$ conformed to the normal distribution; the depth of corrosion pits' evolution law was in the form of double straight lines and the corrosion rate evolution law was in the power function form $\left(y=a \times x^{b}\right)$; and the depth of corrosion pits changed rapidly in the early stage and gradually slowed down in the later stage, while the corrosion rate was just the opposite.
\end{abstract}

Keywords: 7075 aluminum alloy; depth of corrosion pits; corrosion rate; binarization; dynamic law

\section{Introduction}

The 7075 ultra-high-strength aluminum alloy material has a series of advantages such as high specific strength, high specific stiffness, light weight, and so on. It is widely used in national economic construction and the national defense industry, especially in the aviation industry field, in which it plays an irreplaceable role [1,2], such as aircraft wing panel, wing beam, wing rib, and fuselage internal support components [3]. However, with the growth of service time, a series of corrosion problems of 7075 aluminum alloy often occur in the atmospheric environment, especially in coastal areas. According to statistics, most aircraft in coastal areas are in combat readiness on duty (not working) status after being delivered for use, and their stopping time on the ground generally accounts for more than $97 \%$ of the total service time [4,5]. Aluminum alloy materials on the surface of an aircraft airframe will suffer from corrosion damage to varying degrees under adverse conditions such as coastal salt fog, rain water, and humid air, and for the aircraft internal structure, due to its strong concealment, corrosion damage is often more easily ignored and the corrosion threat is greater. A large number of 
studies have shown that aircraft with corrosion damage are more prone to fatigue damage under the action of flight load [6-9], which seriously threatens the flight safety of the aircraft. Therefore, it is very important to analyze and evaluate the corrosion damage of aluminum alloy materials in coastal areas and understand the evolution law of corrosion damage, which is very important to ensure the long-term service safety of aircraft, significantly in coastal islands and reefs.

For the study of corrosion damage law, because outdoor atmospheric corrosion often has a long period and consumes a lot of manpower and resources, laboratory-accelerated corrosion methods are often used. For the accelerated corrosion method in laboratory, two contents need to be clarified. One is the selection of corrosion mode (full immersion, alternate immersion, salt spray, etc.) and the other is to determine the composition and concentration of corrosion medium in the corrosion solution. Because the alternate immersion test can better simulate the process of "alternation of dry and wet" atmospheric corrosion [10] and in order to restore the actual atmospheric corrosion environment more truly, the laboratory-accelerated corrosion method determined in this paper was as follows: Using the alternate immersion corrosion method, the components of various corrosive media, obtained from real monitoring in the Wanning area of Hainan Province (coastal cities in China), were taken as the components of the corrosion solution in this test and the concentration of the corrosion medium monitored was weighed in equal proportion as the concentration of corrosion medium in the accelerated corrosion solution.

As one of the most common corrosion damage forms in the early stage of aluminum alloy structure corrosion, pitting corrosion accounts for the highest proportion in all corrosion types. It does not have obvious corrosion morphology, like denudation, and the corrosion pits on the material surface are easily covered by corrosion products, so it is easy for people to neglect [11]. After pitting corrosion of aluminum alloy, the corrosion pits on the surface of the structure are often the crack source of corrosion fatigue and stress corrosion fracture, which directly affect the residual strength and fatigue life of aluminum alloy structure. Therefore, establishing a reasonable and reliable corrosion pits' evaluation index was the premise of corrosion damage analysis and evaluation. At present, there is no formed evaluation system for corrosion damage (corrosion pits) at home or abroad [12]. The damage tolerance design manual of the U.S. Air Force proposes that "defect influence" should be selected as the most ideal evaluation index for structural corrosion damage. That is to say, the geometric dimension parameters of corrosion damage are important parameters to describe corrosion damage [13]. However, due to the unclear definition of the dangerous corrosion pit, the parameters characterizing the geometric size of corrosion damage will be different [14]. At present, the corrosion pit width [15,16], the aspect ratio $[17,18]$, the projected cross-section area $[19,20]$, and the depth of corrosion pits $[21,22]$ are widely used. However, after corrosion, the corrosion pits' morphology on the surface of the specimen is very complex. If the pit surface is simplified to a regular figure to define its length and width, there will be a big gap with the actual situation. Moreover, the corrosion damage parameters are often of micro scale. How to accurately measure the corrosion damage parameters to reduce the error is also very important. Therefore, in engineering practice, it is necessary to find a simple, accurate, and universal corrosion damage evaluation parameter and, on this basis, the law of accelerated corrosion damage evolution is studied.

After corrosion, the most intuitive evolution characteristics of corrosion pits are the development along the surface and depth direction and the increase of surface roughness. Therefore, two parameters of the depth of corrosion pits $d$ (describing the depth of corrosion damage on the surface of the specimen) and the corrosion rate $C_{R}$ (describing the surface damage degree of the specimen) were introduced as the evaluation indexes of accelerated corrosion damage of 7075 aluminum alloy specimens. According to the real annual average value of atmospheric environmental data in Wanning, Hainan, a new corrosion solution was designed and the traditional alternate immersion corrosion method of using the alternate immersion corrosion test box was improved to simulate the environment of the internal structure of the aircraft. On this basis, the depth of corrosion pits obtained by 3D profile of specimen based on the Rh- 8800 3D video microscope and binarization images based on MATLAB software were proposed, respectively. The optimal linear regression equation combination of the depth of 
corrosion pits was obtained, the dynamic evolution model of the depth of corrosion pits, corrosion rate, and fractal dimension was fitted, and the evolution law of accelerated corrosion damage was analyzed systematically.

\section{Materials and Methods}

\subsection{Materials}

The sample material was 7075-T6 ultra-high-strength aluminum alloy. The reference chemical composition is shown in Table 1. The components of the corrosion solution were $\mathrm{H}_{2} \mathrm{SO}_{4}, \mathrm{HNO}_{3}, \mathrm{NaCl}$, and distilled water. For specific concentrations, see Table 2 in Section 2.2.1.

Table 1. Chemical composition of 7075 aluminum alloy (mass fraction/\%).

\begin{tabular}{ccccccccc}
\hline $\mathbf{S i}$ & $\mathbf{F e}$ & $\mathbf{C u}$ & $\mathbf{M n}$ & $\mathbf{M g}$ & $\mathbf{C r}$ & $\mathbf{Z n}$ & $\mathbf{T i}$ & $\mathbf{A l}$ \\
\hline 0.40 & 0.50 & $1.2 \sim 2$ & 0.30 & $2.1 \sim 2.9$ & $0.18 \sim 0.28$ & $5.1 \sim 6.1$ & 0.20 & allowance \\
\hline
\end{tabular}

The geometric dimension of the test piece was designed according to the provisions of GB / $\mathrm{T}$ 6398-2000. (GB are the first letters of Chinese pinyin "national standard". T means recommendation in Chinese. The same appears below.) The boring and milling process was carried out in the Numerical Control to simulate the detail form of the internal structure of the aircraft wing. In order to remove the burr, after reaming, chamfer no more than $0.3 \times 45^{\circ}$ was made on both sides of the hole to ensure that the hole surface was free of edge fracture and crack. We then selected appropriate processing parameters on the processing machine tool to ensure the surface roughness of the test piece. The surface roughness of the hole was $\mathrm{Ra}=1.6 \mu \mathrm{m}$, which met the technical requirements, as shown in Figure 1.

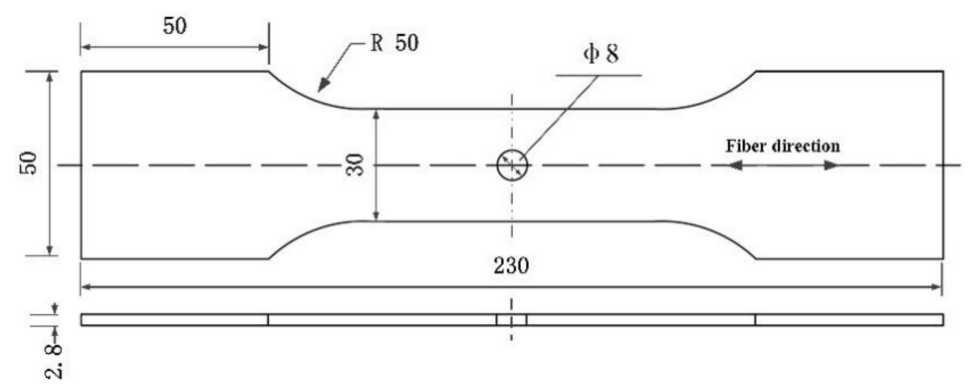

Figure 1. Geometrical dimensions of the test piece.

\subsection{Methods}

\subsubsection{Determination of Corrosion Solution}

The annual average value of atmospheric environmental data obtained from the Hainan, Wanning Natural Environment Test Station through the "China Corrosion and Protection Network" is shown in Table 2.

Table 2. Environmental data in Wanning, Hainan.

\begin{tabular}{ccccccc}
\hline $\begin{array}{c}\text { Environment } \\
\text { Parameters }\end{array}$ & $\begin{array}{c}\text { Relative } \\
\text { Humidity/(\%) }\end{array}$ & $\begin{array}{c}\text { Temperature/ } \\
\left({ }^{\circ} \mathbf{C}\right)\end{array}$ & $\begin{array}{c}\text { Wind Speed/ } \\
(\mathbf{m} / \mathbf{s})\end{array}$ & $\begin{array}{c}\mathrm{NO}_{2} / \\
\left(\mathbf{m g} / \mathbf{m}^{3}\right)\end{array}$ & $\begin{array}{c}\mathrm{SO}_{2} / \\
\left(\mathbf{m g} / \mathbf{m}^{3}\right)\end{array}$ & $\begin{array}{c}\mathrm{Cl}^{-} \text {Deposition Rate/ } \\
\left(\mathbf{m g} / \mathbf{1 0 0} \mathbf{~ c m}^{2} \cdot \mathbf{d}\right)\end{array}$ \\
\hline $\begin{array}{c}\text { Annual } \\
\text { Average }\end{array}$ & 87.6 & 23.9 & 2.43 & $3.03 \times 10^{-3}$ & $0.045 \times 10^{-3}$ & 14.59 \\
\hline
\end{tabular}

The formation of water droplets or water films on the surface of aluminum alloys is a necessary condition for atmospheric corrosion [23]. According to the environmental chemistry of water, the following chemical reactions occur when $\mathrm{SO}_{2}$ and $\mathrm{NO}_{2}$ are dissolved in water: 


$$
\begin{gathered}
\mathrm{SO}_{2}+\mathrm{H}_{2} \mathrm{O} \stackrel{k_{1}}{\rightleftarrows} \mathrm{H}^{+}+\mathrm{HSO}_{3}^{-} \\
2 \mathrm{NO}_{2}(\mathrm{aq})+\mathrm{H}_{2} \mathrm{O} \stackrel{k_{2}}{\rightleftarrows} 2 \mathrm{H}^{+}+\mathrm{NO}_{2}^{-}+\mathrm{NO}_{3}^{-}
\end{gathered}
$$

$\mathrm{SO}_{2}$ and $\mathrm{NO}_{2}$ have their own dissolution equilibrium and obey Henry's law, that is, under isothermal and isobaric conditions, the solubility of a certain gas in the solution is proportional to the equilibrium pressure of the gas on the liquid surface. The solubility in water can be expressed by Formula (3):

$$
\mathrm{X}(\mathrm{aq})=K_{H} \cdot p_{G}
$$

In Formulas (1)-(3) $k_{1}$ is the ionization equilibrium constant, $k_{2}$ is the chemical equilibrium constant, $K_{H}$ is the Henry constant of different gases, and $p_{G}$ is the partial pressure of different gases.

Calculate the solubility of each gas according to Formula (3) and calculate $\mathrm{SO}_{2}$ and $\mathrm{NO}_{2}$ according to ionization balance and chemical balance. Under dissolution equilibrium, equivalent calculations of $\mathrm{H}_{2} \mathrm{SO}_{4}$ and $\mathrm{HNO}_{3}$ with the same $\mathrm{H}^{+}$concentration can obtain their respective molar concentrations. The $\mathrm{Cl}^{-}$deposition is shown in Table 2. The formation and rupture of sea water mist and sea bubbles will produce tiny droplets that undergo a series of evolutions such as cracking and evaporation in the air to form atmospheric salt cores and then continue to deposit on land. Therefore, the influence of the corrosion medium $\mathrm{Cl}^{-}$must be considered. In this paper, it was simplified, and the approximate mass concentration of $\mathrm{NaCl}$ in seawater was $3.5 \%$ as the concentration of $\mathrm{Cl}^{-}$in the accelerated corrosion solution.

According to the abovementioned equal weighting method for the concentration of the corrosion medium, the concentration of each corrosion medium in the accelerated corrosion solution was finally determine. Considering that $\mathrm{Cl}^{-}$is treated according to the mass concentration of seawater, it was already serious. So, only the equivalent corrosion media $\mathrm{H}_{2} \mathrm{SO}_{4}$ and $\mathrm{HNO}_{3}$ of $\mathrm{SO}_{2}$ and $\mathrm{NO}_{2}$ were weighted in equal proportions. The $\mathrm{pH}$ of the accelerated corrosion solution was set to 1 and the corrosion was finally obtained by equivalent weight. The concentration of the medium is shown in Table 3.

Table 3. Medium composition and concentration of accelerated corrosion solution.

\begin{tabular}{ccccc}
\hline Corrosive Medium & Distilled Water & $\mathbf{H}_{2} \mathrm{SO}_{4} /(\mathrm{mol} / \mathrm{L})$ & $\mathbf{H N O}_{3} /(\mathbf{m o l} / \mathrm{L})$ & $\mathrm{NaCl} /(\%)$ \\
\hline Concentration & - & $4.06 \times 10^{-3}$ & $9.19 \times 10^{-2}$ & 3.5 \\
\hline
\end{tabular}

According to general research, it is found that the three ions, $\mathrm{SO}_{4}{ }^{2-}, \mathrm{NO}_{3}{ }^{-}$, and $\mathrm{Cl}^{-}$, have the greatest influence on the atmospheric corrosion of aluminum alloys [24,25]. So, it was reasonable to introduce these three ions in the accelerated corrosion solution of this experiment. The $\mathrm{Cl}^{-} / \mathrm{NO}_{3}{ }^{-}$was 6.7465, very close to 6.667 in the standard EXCO corrosion solution (EXCO corrosion solution is the standard solution for accelerated corrosion test of 7xxx series high strength aluminum alloy).

2.2.2. A Method of Alternate Immersion Corrosion for Simulating the Environment of the Internal Structure of the Aircraft

For the alternate immersion corrosion test, the current traditional method was accelerated corrosion through the alternate immersion corrosion test box. However, through pretests, this paper found that when controlling the humidity in the drying stage, the corrosion test box used air with a certain humidity to be transferred to the test environment, which obviously increased the evaporation rate of the liquid film on the surface of the test piece. In the Wanning area, the true corrosion environment of the internal structure of the aircraft with high humidity and low wind speed (almost none) has no small deviation. In order to better solve this problem, this article adopted the method of saturated salt solution to generate a constant humidity environment, avoiding the flow of air in the environment, which is closer to the corrosive environment of the interior structure of indoor aircraft in Wanning, Hainan. 
Since there is no corrosion test box for controlling humidity by saturated salt solution, this test was conducted by manual method. First, we placed the test piece in the liquid tank for immersion by artificial immersion. After reaching the specified immersion time, we removed the sample and placed it in the electric constant temperature drying box. The bottom of the closed container in the drying box contained about one-third of the total volume of saturated $\mathrm{KCl}$ salt solution. The upper space of the salt solution was an environment with constant relative humidity, and the temperature was set to 23.9 ${ }^{\circ} \mathrm{C}$. At this temperature, in the literature [26,27], the saturated $\mathrm{KCl}$ salt solution produces a constant humidity environment with relative humidity ( $\mathrm{RH} \%$ ) between $84-85 \%$, while the measured relative humidity of the humidity environment on the upper side of the closed container was $88 \%$, which is very close to the average humidity of $87.6 \%$ in Wanning, Hainan. The error did not exceed $0.5 \%$ and it had good simulation. We carried out the cycle in turn. The process is shown in Figure 2. According to the alternate immersion test standard for metals and alloys (GB/T 19746-2018), the surface area ratio of the test piece in the corrosion tank and the accelerated corrosion solution was $30 \mathrm{~mL} / \mathrm{cm}^{2}$. The $\mathrm{pH}$ value of the corrosion solution was measured every other day. When the $\mathrm{pH}$ changed more than 0.2 , we increased the acid solution, according to the ratio of $\mathrm{H}_{2} \mathrm{SO}_{4}$ and $\mathrm{HNO}_{3}$ in Table 3, to restore and adjust the $\mathrm{pH}$ value to ensure that the corrosion environment in the entire process of the accelerated corrosion test in the laboratory remained consistent.
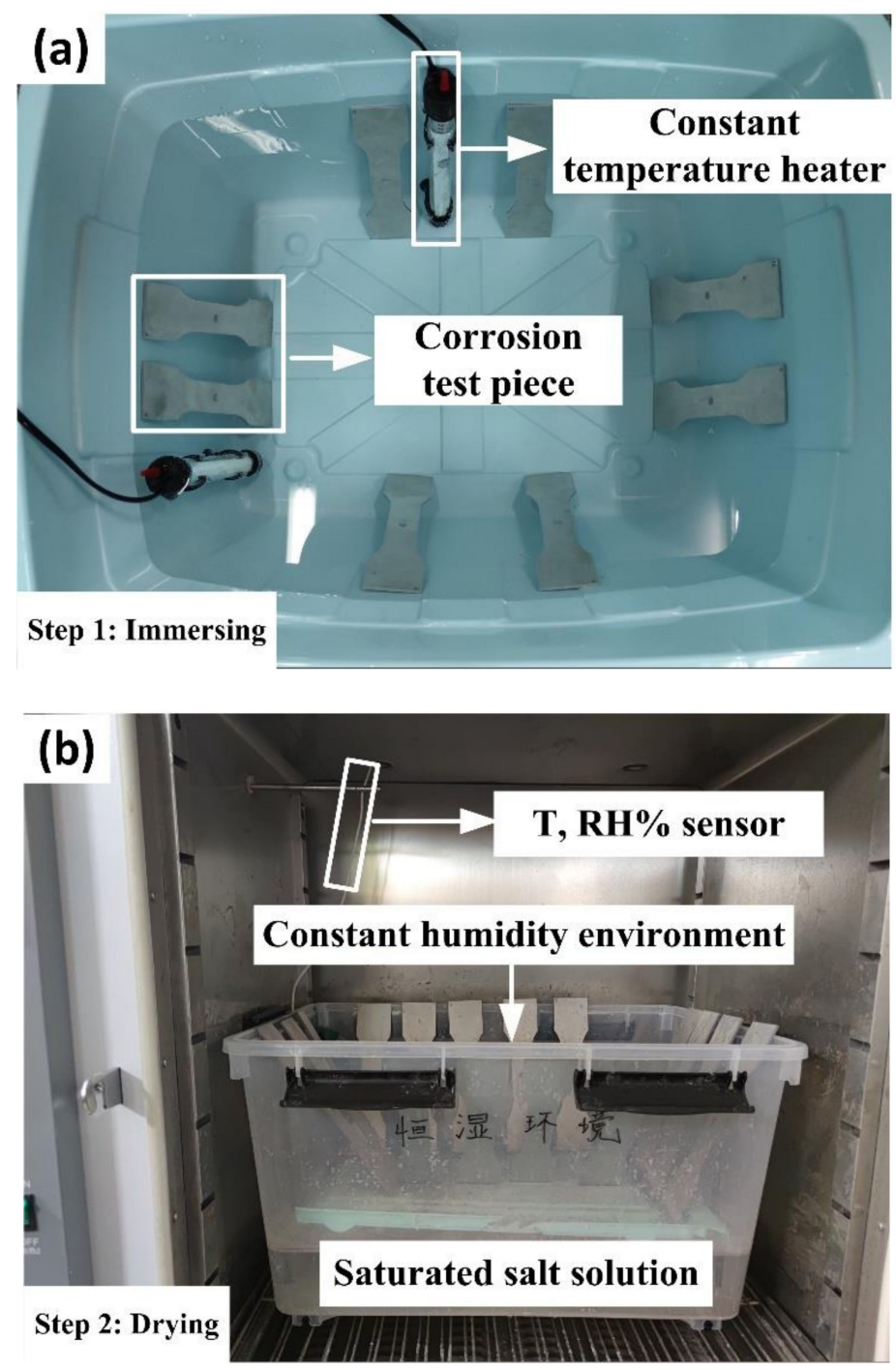

Figure 2. Artificial alternate immersion corrosion test. (a) Immersing period, (b) drying period. 
Because the internal structure of the aircraft avoids sunlight, wind, and rain, it is closer to the indoor ventilated atmospheric environment than the outdoor atmospheric exposure environment. So, the corrosion environment spectrum did not consider the time of rainfall. Corrosion environment spectrum is shown in Figure 3. The fog and dew weather action time and the total annual time of 7:53 in the atmospheric environment of Wanning, Hainan, were selected as the ratio of the immersion test piece and the drying time in the alternate immersion experiment. The alternate immersion cycle of this experiment was set to $8 \mathrm{~h}$, that is, $0.94 \mathrm{~h}$ immersing and $7.06 \mathrm{~h}$ drying (7:53). The actual annual average temperature of Wanning, Hainan, of $23.9^{\circ} \mathrm{C}$ and the average annual relative humidity of $87.6 \%$ were selected as the test environment during drying. After 8 h, 24 h, 48 h, 72 h, 96 h, 120 h, 144 h, 192 h, and $240 \mathrm{~h}$, respectively, the test pieces were taken out in sequence, rinsed with distilled water, dried, and finally stored in a drying dish.

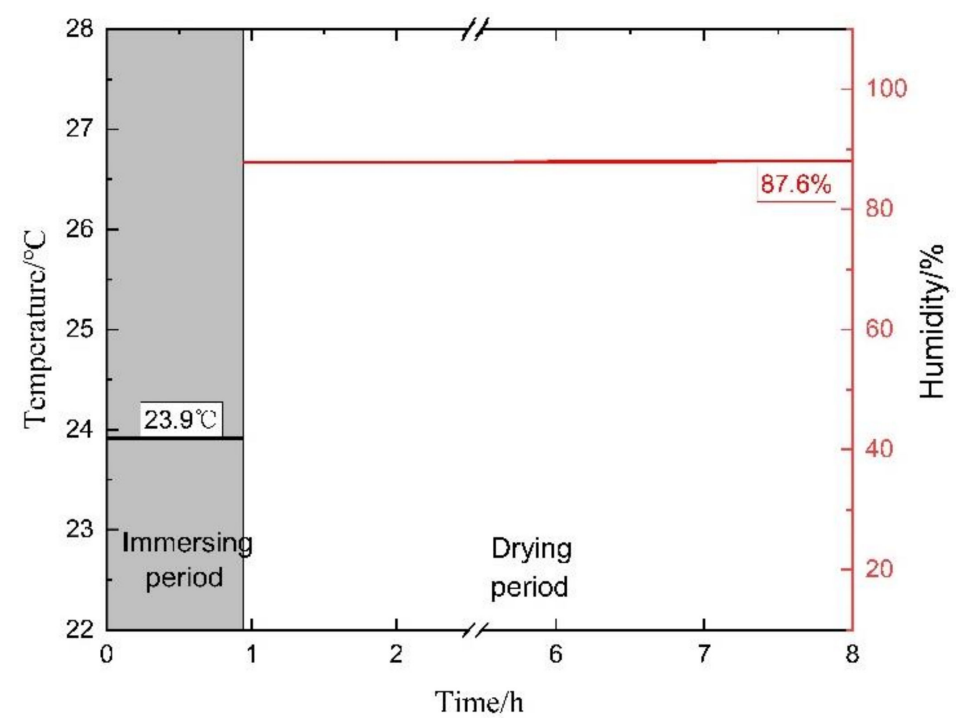

Figure 3. Accelerated corrosion environment spectrum.

\section{Results and Discussion}

\subsection{Corrosion Evaluation}

The macroscopic corrosion morphology of the specimen under different corrosion times is shown in Figure 4. From the macroscopic morphology, it can be clearly seen that as the corrosion time continued to increase, the corrosion type gradually changed from pitting to denudation after $120 \mathrm{~h}$.

In the early stage of corrosion, when the accelerated corrosion time was $8 \mathrm{~h}$, the surface gloss of the specimen was good and the corrosion pits were few and relatively uniform. When the accelerated corrosion time was $24 \mathrm{~h}$ and $48 \mathrm{~h}$, there were corrosion pits visible to the naked eye, the pit density increased, and the color gradually darkened, but the surface of the specimen still had metallic luster. When the accelerated corrosion time was $72 \mathrm{~h}$ and $96 \mathrm{~h}$, the specimen had basically lost its metallic luster. When the accelerated corrosion time was $120 \mathrm{~h}$, the corrosion pits on the surface of the specimens had been aggregated into large areas, the small pits were connected to each other to form large pits, and the surface roughness of the specimens was further increased. Through further observation by VEGA II XMU scanning electron microscope (TESCAN, Shanghai, China), it was found that several micro cracks along intergranular corrosion appeared in the direction of the plane (as shown in Figure 5).

In the denudation stage, when the accelerated corrosion time was $144 \mathrm{~h}$, the surface of the specimen began to show obvious erosion characteristics: Bulge. When the accelerated corrosion was $192 \mathrm{~h}$, the erosion phenomenon was more obvious. From the macroscopic point of view, we saw that there were a lot of scales and flakes on the surface of the specimen. Figure 6 shows the 3D morphology of the specimen surface, which can be seen that the surface of the specimen has a "cliff-type" shape due to 
the peeling of the material. It indicates that the phenomenon of denudation and stratification occurred. When the corrosion was accelerated for $240 \mathrm{~h}$, the whole exfoliation layer appeared on the surface of the specimen. Since the damage evolution law of pitting stage (corrosion pits) was mainly studied in this paper and it was difficult to accurately obtain the depth of corrosion pits' data after denudation, the corrosion cycle before $120 \mathrm{~h}$ was selected for the following analysis.
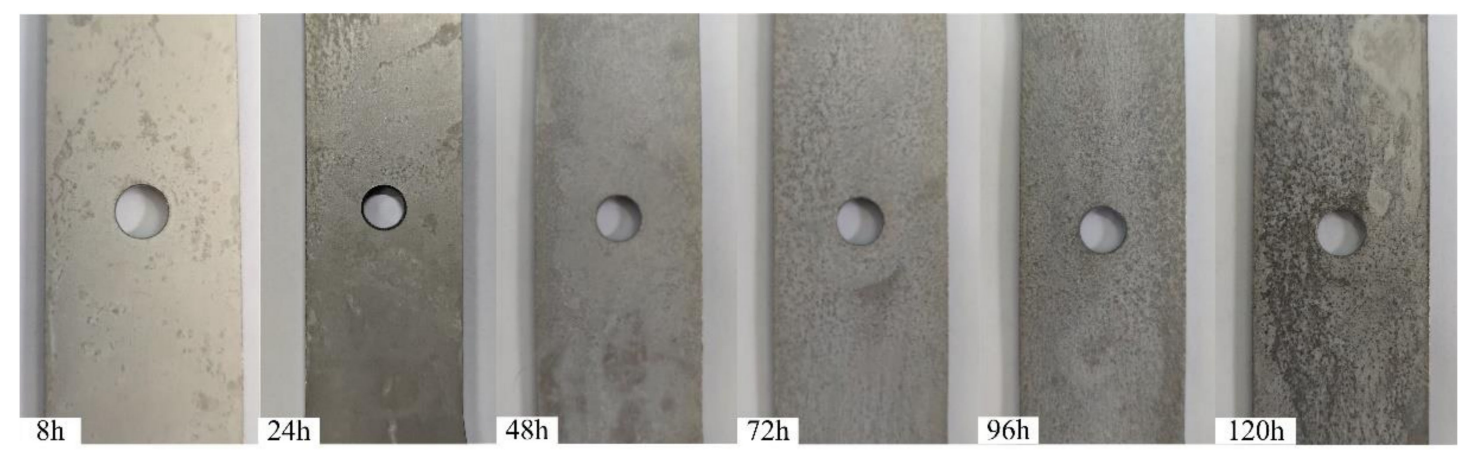

(a)

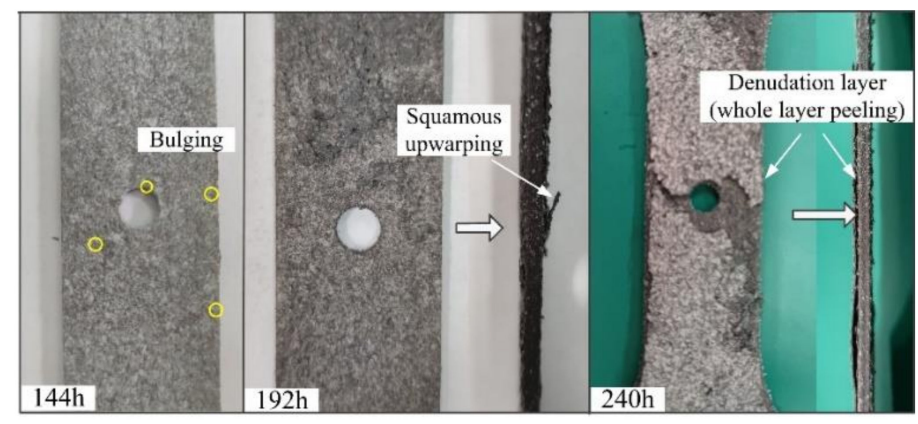

(b)

Figure 4. Corrosion morphology. (a) Pitting, (b) denudation.

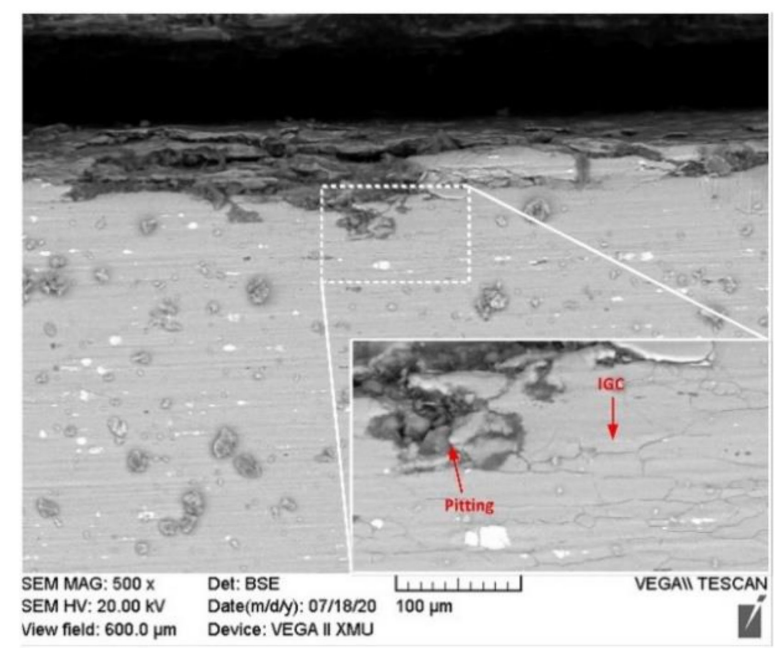

Figure 5. Micro morphology of longitudinal section $(t=120 \mathrm{~h})$. 


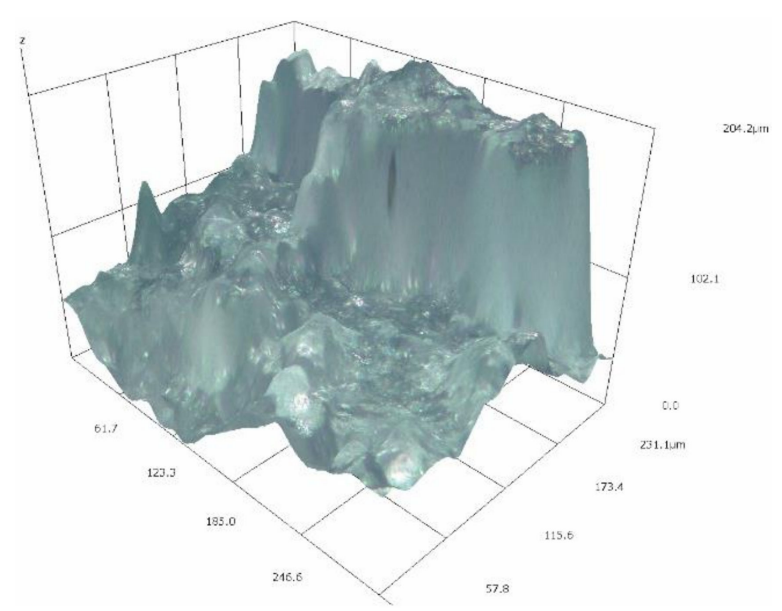

Figure 6. 3D topography of surface $(t=192 \mathrm{~h})$.

\subsection{Depth of Corrosion Pits}

\subsubsection{Measurement Method}

After accelerated corrosion in the laboratory, the surface shape of the test piece was complex and the contour line was uneven. In order to obtain the depth of the corrosion pits, 3D imaging technology can be used to automatically identify the height of the designated part of each pit area, and then obtained by the height difference between the bottom of the pit and the top of the pit. However, because this method cannot accurately lock the height of the bottom of each pit and because of the unevenness of the corrosion area and the overall thinning of the thickness of the specimen, the measurement of the top area of the corrosion pits is often selected too high due to subjectivity.

The cross-sectional contour of the corrosion area can clearly reflect the detailed characteristics of the corrosion area in the depth direction, and the height difference between the bottom and the top of the corrosion pit can be accurately obtained. Therefore, this paper first used the Rh-8800 3D video microscope (Hirox, Tokyo, Japan) to obtain the three-dimensional morphology of the corrosion specimen at a magnification of 1200 times. We then intersected the section perpendicular to the length of the specimen with the three-dimensional image and then obtained the contour line information of several sections in the $x$ direction of the specimen, as shown in Figure 7.
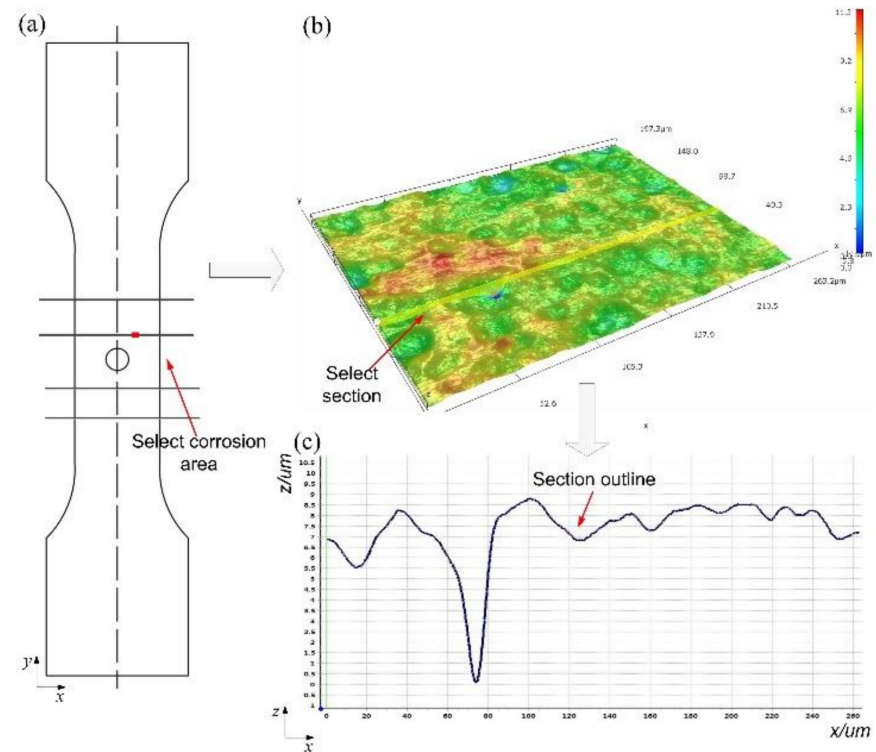

Figure 7. Obtaining the depth of corrosion pits. (a) Section selected for the specimen; (b) Three-dimensional morphology of the corrosion specimen; (c) Schematic diagram of section contour. 
According to the above method, the depth of corrosion pits at different corrosion times in the pitting phase $(t \leq 120 \mathrm{~h})$ was measured. To ensure the reliability of the data, three test pieces were measured for each corrosion cycle and 15 areas were measured for each test piece. We calculated the average of the depth of corrosion pits under each corrosion time. The results are shown in Figure 8.

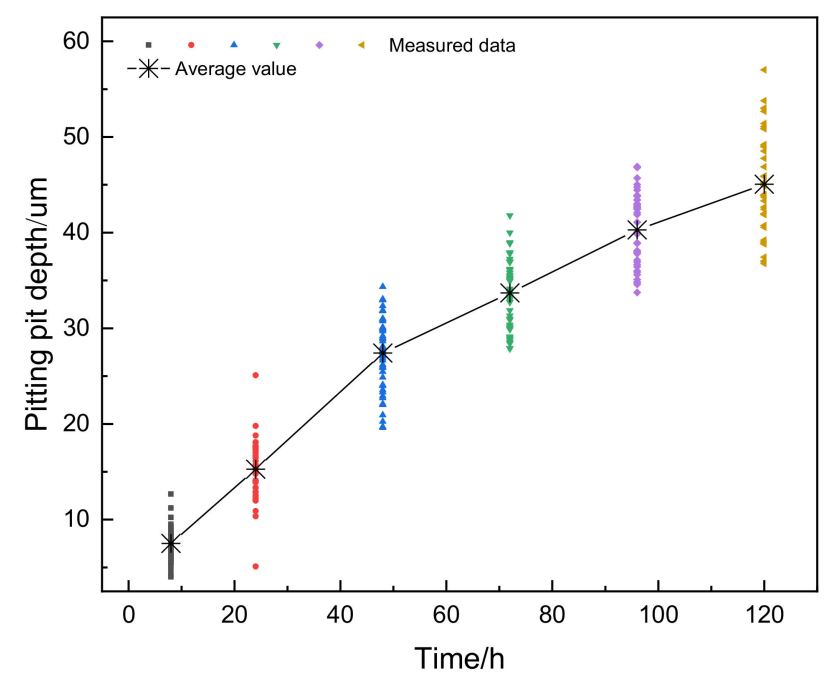

Figure 8. The depth of corrosion pits under different corrosion time.

\subsubsection{Distribution Law}

According to the research results, the distribution forms of the depth of corrosion pits of aluminum alloy materials generally included Gumbel (type I maximum) distribution, normal distribution, lognormal distribution, and two-parameter Weibull distribution [28-30]. In this section, statistical methods were applied to analyze the depth of corrosion pits, and linear regression equation fitting was conducted for the four distribution forms.

When the significance level is $\alpha=0.05$, that is, within the confidence level of 0.95 , the linear correlation critical value of each corrosion cycle satisfying the assumed distribution can be solved by Formula (4).

$$
r_{C}=\frac{t_{\alpha}(n-1)}{\sqrt{n-2+t_{\alpha}^{2}(n-1)}}
$$

The number $\mathrm{N}$ of corrosion pits selected for each corrosion time was 45 , which resulted in $t_{0.05}$ $(45-2)=1.681$ and $t_{0.05}(45-1)=1.680$, and the linear correlation critical value $r_{C}$ was calculated to be 0.248 .

It can be seen from Table 4 that the correlation coefficients of the four distributions were much greater than the critical coefficient $r_{C}$. It was obtained that, under accelerated corrosion conditions in the laboratory, the depth of corrosion pits of the specimens with different corrosion times could better follow the normal distribution and the Gumbel distribution, lognormal distribution, and two-parameter Weibull distribution. Further comparing the advantages and disadvantages of the three distributions, we obtained that, under different corrosion times, when the corrosion time was $8 \mathrm{~h}$, the absolute value of the correlation coefficient of the Gumbel distribution was 0.991 (the highest) and when the corrosion time was $24 \mathrm{~h}$, the Weibull distribution of the correlation coefficient was 0.939 (the highest). So, the best distributions of the first two corrosion cycles were the Gumbel distribution and the two-parameter Weibull distribution. When the corrosion time was $48 \mathrm{~h}, 72 \mathrm{~h}, 96 \mathrm{~h}$, and $120 \mathrm{~h}$, the correlation coefficients of the normal distribution were above 0.990 , better than the other three distributions. So, the optimal distribution was the normal distribution. 
Table 4. Fitting results of the depth of corrosion pits' values corresponding to three different distribution parameters.

\begin{tabular}{cccccccc}
\hline Distribution Type & Parameter & $\mathbf{8} \mathbf{h}$ & $\mathbf{2 4} \mathbf{h}$ & $\mathbf{4 8} \mathbf{h}$ & $\mathbf{7 2} \mathbf{~ h}$ & $\mathbf{9 6} \mathbf{h}$ & $\mathbf{1 2 0} \mathbf{~}$ \\
\hline \multirow{2}{*}{ Normal distribution } & $\mu$ & 7.352 & 15.272 & 27.414 & 33.698 & 40.291 & 45.064 \\
$\left(\mathrm{Y}_{1}\right)$ & $\sigma$ & 1.749 & 3.043 & 4.053 & 3.623 & 3.741 & 5.120 \\
& $r$ & 0.957 & 0.909 & 0.990 & 0.991 & 0.994 & 0.983 \\
\hline \multirow{2}{*}{ Gumbel distribution } & $\mu$ & 6.892 & 13.991 & 26.072 & 31.891 & 38.021 & 44.123 \\
$\left(\mathrm{Y}_{2}\right)$ & $\sigma$ & 2.312 & 3.221 & 4.121 & 3.458 & 3.889 & 4.451 \\
& $r$ & -0.991 & -0.935 & -0.939 & -0.967 & -0.959 & -0.971 \\
\hline \multirow{2}{*}{ Lognormal distribution } & $\mu$ & 1.967 & 2.703 & 3.222 & 3.512 & 3.692 & 3.846 \\
$\left(\mathrm{Y}_{3}\right)$ & $\sigma$ & 0.238 & 0.233 & 0.167 & 0.108 & 0.0933 & 0.108 \\
& $r$ & 0.968 & 0.837 & 0.978 & 0.990 & 0.989 & 0.981 \\
\hline \multirow{2}{*}{ Weibull distribution } & $\beta$ & 4.362 & 5.261 & 7.556 & 10.302 & 12.120 & 9.812 \\
$\left(\mathrm{Y}_{4}\right)$ & $\sigma$ & 8.036 & 16.438 & 27.105 & 35.320 & 41.990 & 49.377 \\
& $r$ & 0.980 & 0.939 & 0.982 & 0.960 & 0.961 & 0.959 \\
\hline
\end{tabular}

\footnotetext{
Note: In the table, $\mu$ is the position parameter, $\sigma$ is the scale parameter, $\beta$ is the shape parameter, and $r$ is the correlation coefficient.
}

Through the above analysis, we statistically obtained the optimal distribution linear regression equation combination corresponding to the depth of corrosion pits under different corrosion times, as shown in Table 5.

Table 5. Combination of optimal linear regression equations for the depth of corrosion pits.

\begin{tabular}{ccc}
\hline Corrosion Time $\boldsymbol{h}$ & Distribution Form & Linear Regression Equation \\
\hline 8 & Gumbel distribution & $\mathrm{Y}_{2}=-4.569-0.698 d$ \\
24 & Weibull distribution & $\mathrm{Y}_{4}=-13.987+4.966 \ln d$ \\
48 & Normal distribution & $\mathrm{Y}_{1}=-144.350+7.089 d$ \\
72 & Normal distribution & $\mathrm{Y}_{1}=-217.591+7.940 d$ \\
96 & Normal distribution & $\mathrm{Y}_{1}=-260.837+7.715 d$ \\
120 & Normal distribution & $\mathrm{Y}_{1}=-194.385+5.590 d$ \\
\hline
\end{tabular}

The equations and experimental data in Table 5 are plotted in Figure 9. From the figure, it can also be seen that the linear regression equation combination under different corrosion times fitted in this paper can better reflect the distribution law of the depth of corrosion pits.

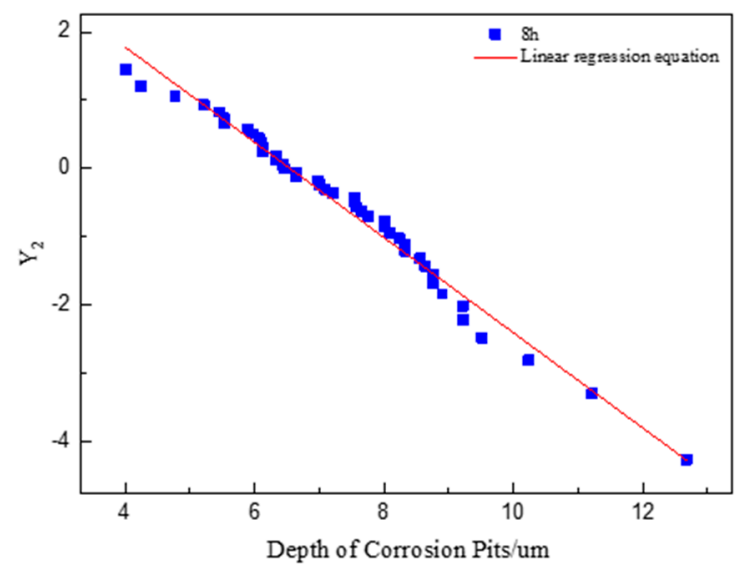

(a)

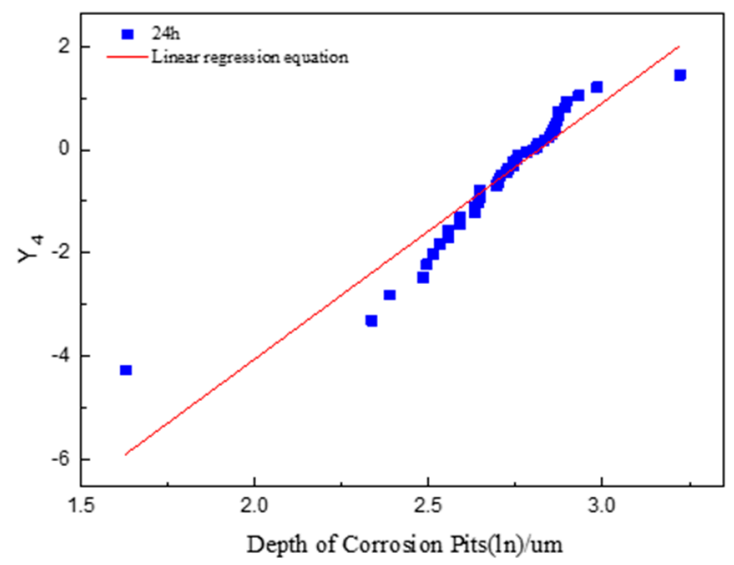

(b)

Figure 9. Cont. 


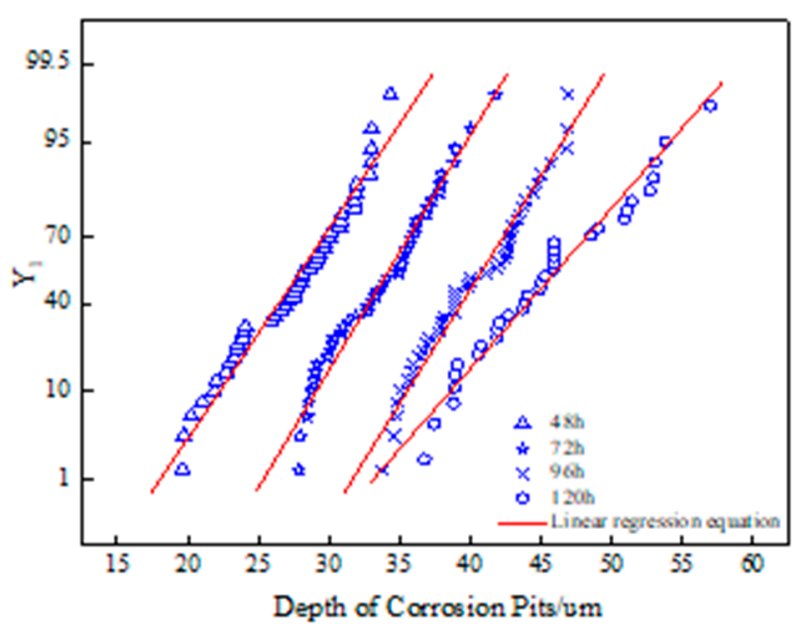

(c)

Figure 9. Fitting result of optimal distribution type of the depth of corrosion pits. (a) Corrosion time $t$ $=8 \mathrm{~h},(\mathbf{b})$ corrosion time $t=24 \mathrm{~h},(\mathbf{c})$ corrosion time $t=48 \mathrm{~h}, 72 \mathrm{~h}, 96 \mathrm{~h}$, and $120 \mathrm{~h}$.

\subsubsection{Dynamic Evolution Model}

Fitting the average depth of the corrosion pits and the corrosion time by the ORIGIN 2018 software (OriginLab, Northampton, MA, USA), the dynamic evolution model of the depth of the corrosion pits was obtained in the form of a double line, as shown in Formulas (5) and (6).

$$
\begin{gathered}
y=1.557+0.550 x x=(0,24) \\
y=15.778+0.248 x x=(24,120)
\end{gathered}
$$

In the formulas, $y$ is the depth of the corrosion pits, $x$ is the corrosion time, and the correlation between the two fitting curves is above 0.99, as shown in Figure 10. The obtained bilinear evolution form is also consistent with the conclusions of most current corrosion research reports [31]. Combining the image change rule and the corrosion mechanism, it was not difficult to find that, in the early stage of corrosion, there were fewer corrosion products and the corrosion proceeded faster in the depth direction. As the corrosion time increased, the corrosion products accumulated in the corrosion pit, according to the negative in the chemical reaction. Feedback adjustment slowed down the corrosion rate, and the rate of change of the depth of corrosion pits had a tendency to gradually decay.

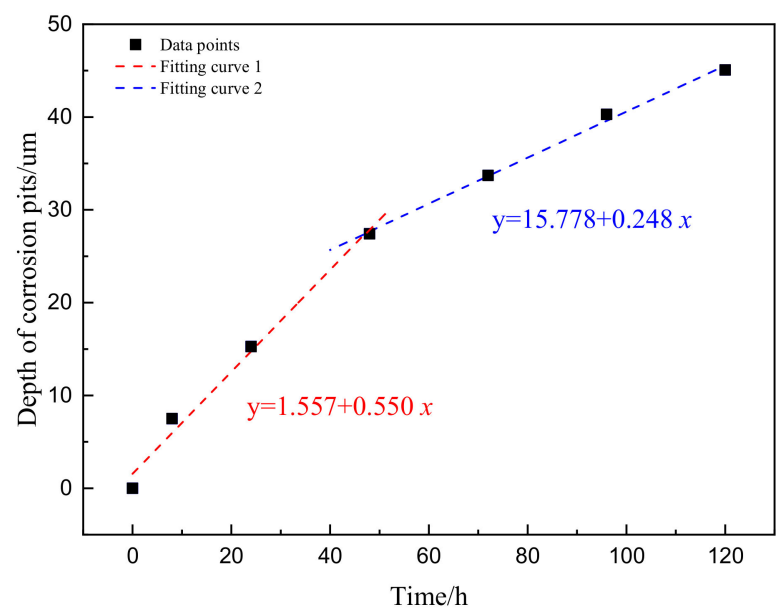

Figure 10. Dynamic model of the depth of corrosion pits' dynamics. 


\subsection{Corrosion Rate}

With the increase of the corrosion time, the corrosion pits area gradually increased in addition to the depth direction. So, by introducing the corrosion rate $C_{R \text {, we }}$ can effectively reflect the severity of corrosion, which is defined as Formula (7):

$$
C_{R}=\frac{1}{A} \sum_{i=1}^{n} A_{i} \times 100 \%
$$

In the formula, $A$ represents the surface area of the selected corrosion area (projected area), $n$ is the number of corrosion pits $(I=1,2, \ldots, n)$, and $A_{i}$ is the surface area of the $i$-th corrosion pit (projected area).

\subsubsection{Image Binarization}

For the acquisition of corrosion rate, the most important thing is to be able to accurately obtain the surface area of corrosion pits, but the corrosion morphology is often more complicated. Even under a high-power microscope, it is difficult to accurately lock the morphology of the pits. If it is equivalent to a regular figure or judged by the naked eye, it will inevitably lead to large errors due to subjectivity. In order to accurately extract the corrosion pits' shape on the corroded surface and obtain the dynamics of the corrosion rate, this paper proposes a method to obtain the corrosion rate based on image binarization. The specific implementation process is shown in Figure 11.

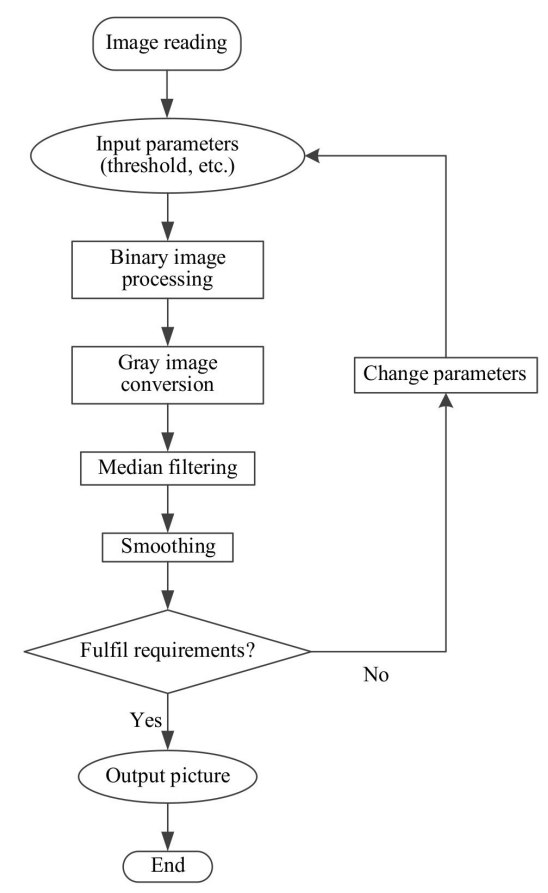

Figure 11. Flow chart of binarization processing of corrosion pits' image.

Through the binarization processing of the corrosion morphology based on MATLAB R2014a software (MathWorks, Natick, MA, USA), the binarization images at different corrosion times were obtained, as shown in Figure 12. The scale bar is 1:1400. There are only two colors in the image, black and white, which represent the substrate and corrosion pits, respectively.

After the image was binarized, the corrosion rate could be easily solved according to the pixel value represented by the pit and the aggregate size of the original image. In this paper, three test pieces were selected under each corrosion time, and a total of four sections were selected for each test piece. The results are shown in Figure 13. 


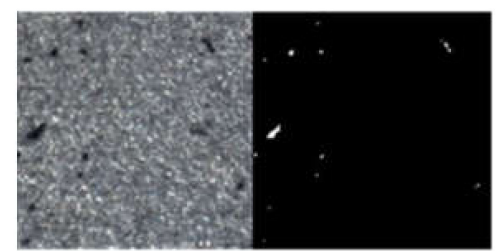

(a)

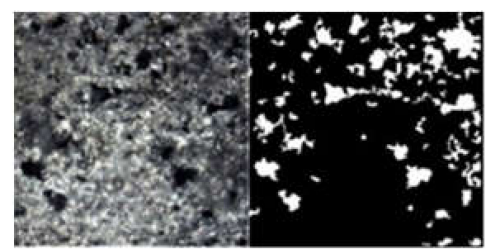

(d)

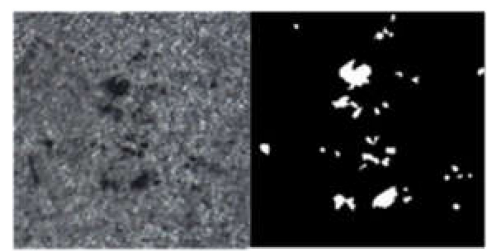

(b)

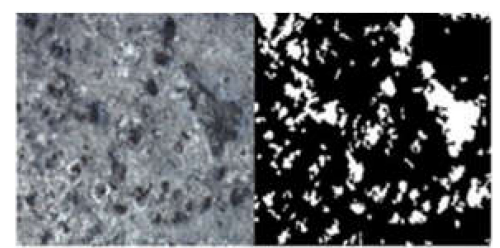

(e)



(c)

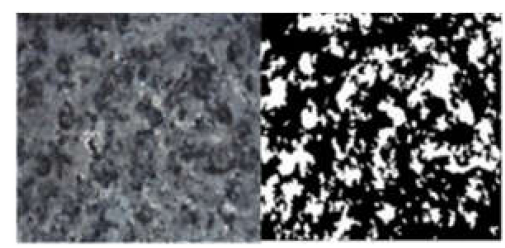

(f)

Figure 12. Binarization morphology of the specimen under various corrosion times. (a) Corrosion time $\mathrm{t}=8 \mathrm{~h},(\mathbf{b})$ corrosion time $\mathrm{t}=24 \mathrm{~h},(\mathbf{c})$ corrosion time $t=48 \mathrm{~h},(\mathbf{d})$ corrosion time $t=72 \mathrm{~h},(\mathbf{e})$ corrosion time $t=96 \mathrm{~h}$, (f) corrosion time $t=120 \mathrm{~h}$.

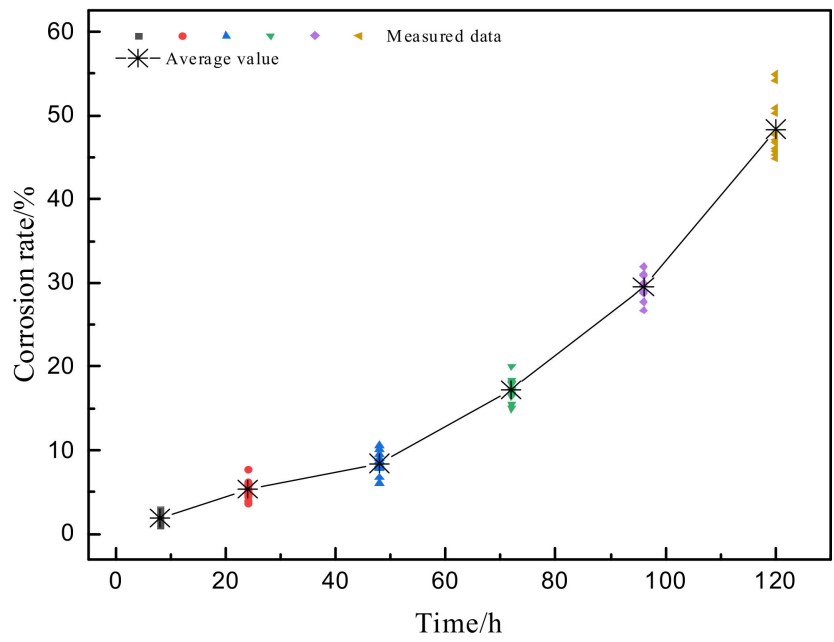

Figure 13. Corrosion rate under different corrosion times.

\subsubsection{Dynamic Evolution Model}

Fitting the relationship between the corrosion rate and corrosion time by the ORIGIN software, the obtained dynamic evolution model was in the form of power function. As shown in Formula (8), the fitting correlation coefficient was 0.989 , which had a good correlation.

$$
y=0.00476 \times x^{1.923}
$$

From the curve obtained by fitting (Figure 14), it can be seen that the pitting corrosion rate increased with the increase of the corrosion time. In the early stage of corrosion, the corrosion rate of the pit increased slowly. After $48 \mathrm{~h}$, the slope of the curve increased, indicating that the corrosion rate increased and accelerated. Putting the dynamic evolution curves of the depth of corrosion pits and corrosion rate in Figure 15 for comparison, it was found that in the early stage of corrosion, the depth of corrosion pits grew faster and the corrosion pits' area increased more slowly. The accumulation of the corrosion pits slowed down the growth of the corrosion pits in the depth direction. The rate of change of the corrosion pits' areas increased and combined with each other, and gradually developed into more serious corrosion. 


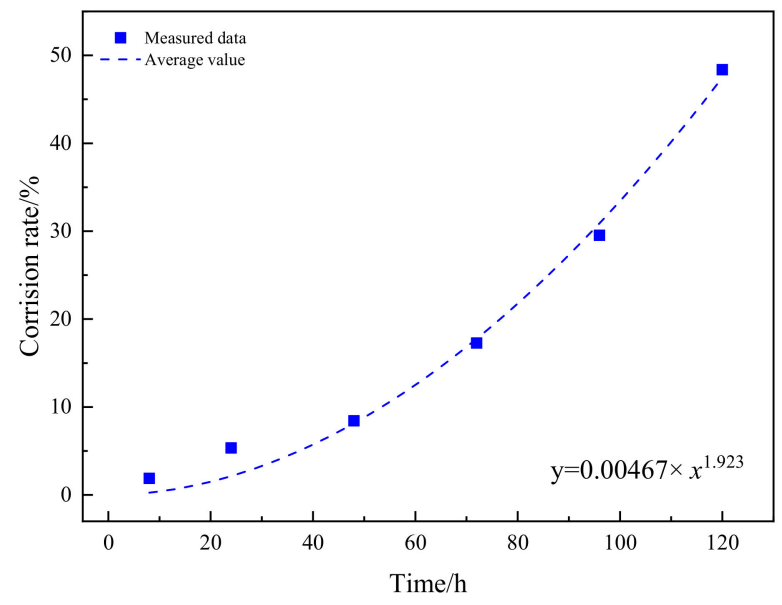

Figure 14. Dynamic model of corrosion rate dynamics.

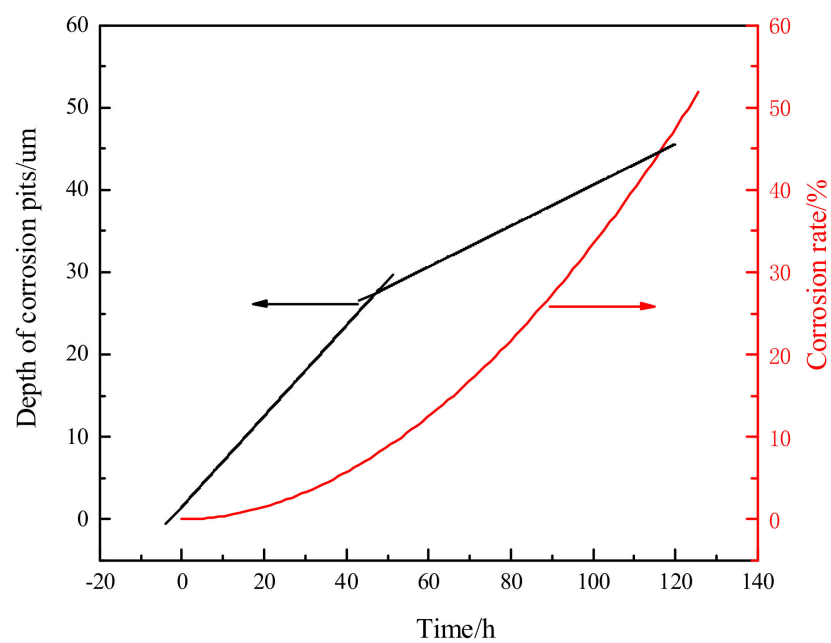

Figure 15. Comparison chart of the depth of corrosion pits and corrosion rate change trend.

By comparing the dynamic evolution curves of the depth of corrosion pits and corrosion rate in Figure 15, it was found that in the initial stage of corrosion, the depth of corrosion pits increased rapidly and the pit area increased slowly, but it was just the opposite in the later stage. This is because in the early stage of corrosion, the corrosion products in the corrosion pits gradually accumulated and the chemical reaction products increased, thus inhibiting the reaction rate. In the later stage, due to the catalytic mechanism of $\mathrm{Cl}^{-}, \mathrm{Cl}^{-}$outside the corrosion pits was continuously enriched in the pit and was also enriched in the area without corrosion pits, which made $\mathrm{Cl}^{-}$accumulate and nucleate in the area, thus leading to the anode addition in the area. With rapid dissolution, the corrosivity increased continuously, the corrosion pit area began to increase continuously, the corrosion rate rose rapidly, and the corrosion pits became a piece.

\section{Conclusions}

(1) According to the characteristics of the atmospheric environment in Wanning, Hainan, a corrosion solution was designed and the traditional alternate immersion corrosion method of using the alternate immersion corrosion test box was improved to simulate the environment of the internal structure of the aircraft.

(2) According to the results of statistical analysis, the depth of corrosion pits can well obey Gumbel distribution, normal distribution, lognormal distribution, and two-parameter Weibull distribution. When the accelerated corrosion time was $8 \mathrm{~h}$ and $24 \mathrm{~h}$, the optimal distribution model was Gumbel 
distribution and Weibull distribution, respectively. When the accelerated corrosion time was $48 \mathrm{~h}$, $72 \mathrm{~h}, 96 \mathrm{~h}$, and $120 \mathrm{~h}$, the optimal distribution model was normal distribution.

(3) The proposed image binarization method and 3D profile recognition method can accurately and conveniently reflect the morphology characteristics of the corrosion pits and quantitatively calculate the depth of corrosion pits and corrosion rate. Compared with the traditional estimation methods, the proposed method had great improvement and had strong engineering value.

(4) The dynamic model of the depth of corrosion pits and corrosion rate evolution was obtained. Through comparison, it was found that the corrosion damage was mainly reflected in the increase of the depth of corrosion pits in the early stage of corrosion, and the growth rate of the pitting corrosion rate was relatively slow. After $48 \mathrm{~h}$, the growth rate of the depth of corrosion pits began to slow down, while the growth rate of pitting corrosion rate accelerated.

Author Contributions: Z.G.: Experiment planning, experiment measurements, data analysis, and manuscript preparation. Y.H.: Experiment planning. S.Z.: Experiment measurements. T.Z. and F.Y.: Data analysis. All authors have read and agreed to the published version of the manuscript.

Funding: This research was funded by National Defense Pre-research Foundation (61409220202).

Conflicts of Interest: The authors declare no conflict of interest.

\section{References}

1. Chantzis, D.; Van-Der-Veen, S.; Zettler, J. An industrial workflow to minimise part distortion for machining of large monolithic components in aerospace industry. Procedia CIRP 2013, 8, 281-286. [CrossRef]

2. Chen, Z.; Zheng, Y.; Zhu, C. Effect of pre-stretching on geometric accuracy and mechanical properties of 7075 aluminum alloy plats. Nonferrous Met. Sci. Eng. 2019, 10, 40-47.

3. Gurgen, S.; Sackesen, I.; Kushan, M.C. Fatigue and corrosion behavior of in-service AA7075 aircraft component after thermo-mechanical and retrogression and re-aging treatments. Proc. Inst. Mech. Eng. 2019, 233, 1764-1772. [CrossRef]

4. Zhao, P.F.; Su, X.Q.; Wu, J.S. Accelerated corrosion test spectrum of typical reef atmospheric environment. Equip. Environ. Eng. 2019, 16, 14-21.

5. Cui, T.F. Effect of Stress and Egalvanic Factors on the Durability of Aluminum Alloy; Northwestern Polytechnical University: Xi'an, China, 2017; pp. 41-49.

6. Brooks, C.L.; Simpson, D. Integrating real time age degradation into the structural integrity process. In Proceedings of the RTO Meeting Proceedings, Corfu, Greece, 7-9 October 1998; pp. 195-207.

7. Chlistovsky, R.M.; Heffernan, P.J.; Duquesnay, D.L. Corrosion-fatigue behaviour of 7075-T651 aluminum alloy subjected to periodic overloads. Int. J. Fatigue 2007, 29, 1941-1949. [CrossRef]

8. Li, X.; Mu, Z.; Jia, M. Effect of Loading Frequency on Corrosion Fatigue Crack Growth Rate of Aerospace Aluminum Alloy. Mater. Mech. Eng. 2014, 38, 50-52, 98. [CrossRef]

9. Ye, Z.Y.; Liu, D.X.; Zhang, X.H. Corrosion Fatigue Behavior of 7A85 Aluminum Alloy Thick Plate in NaCl Solution. Acta Metall. Sin. Engl. Lett. 2015, 28, 1047-1054. [CrossRef]

10. Dan, Z.; Takigawa, S.; Muto, I. Applicability of constant dew point corrosion tests for evaluating atmospheric corrosion of aluminium alloys. Corros. Sci. 2011, 53, 2006-2014. [CrossRef]

11. Pao, P.S.; Gill, S.J.; Feng, C.R. On fatigue crack initiation from corrosion pits in 7075-T7351 aluminum alloy. Scr. Mater. 2000, 43, 391-396. [CrossRef]

12. Miedlar, P.C.; Berens, A.P.; Gunderson, A. USAF Damage Tolerant Design Handbook: Guidelines for the Analysis and of Damage Tolerant Aircraft Structure; AFRL-VA-WP-TR-2003-3002; University of Dayton Research Institute: Dayton, OH, USA, 2002.

13. Zhang, Y.H. The Corrosion Damage and Its Effect e on Life of Aircraft Structure; Northwestern Polytechnical University: Xi'an, China, 2007.

14. Sun, L. The Study on Corrosion Morphology and Residual Fatigue Life of Corroded Aluminium Alloy; Nanjing University of Aeronautics and Astronautics: Nanjing, China, 2013.

15. Ren, K.L.; Lv, G.Z.; Zhang, Y.H. The correlation between corrosion pit with equivalent initial surface crack. Struct. Environ. Eng. 2006, 3, 50-57. 
16. DuQuesnay, D.L.; Underhill, P.R.; Britt, H.J. Fatigue crack growth from corrosion damage in 7075-T6511 aluminum alloy under aircraft loading. Int. J. Fatigue 2003, 25, 371-377. [CrossRef]

17. Yu, M.F. Finite Element Simulation of the Influence of 20CrMnTi Pitting Corrosion on Stress Concentration; Guizhou University: Guizhou, China, 2018.

18. Walde, K.; Hillberry, B.M. Characterization of pitting damage and prediction of remaining fatigue life. Int. J. Fatigue 2008, 30, 106-118.

19. Murakami, Y. Metal Fatigue: Effects of Small Defects and Nonmetallic Inclusions; Elsevier: Amsterdam, The Netherlands, 2002.

20. Adjel, S.; Merakeb, N.; Benchouia, S. Effect of corrosion pit density on the fatigue life of aluminum 1050A. Int. J. Adv. Manuf. Technol. 2018, 97, 3163-3177. [CrossRef]

21. Ye, B.Y.; Zhu, F.; Xu, H.B. Probability Model Study on Corrosion Damage of Aircraft Structure. Aviat. Maint. Eng. 2019, 7, 36-37.

22. Silva, J.W.J.; Bustamante, A.G.; Codaro, E.N. Morphological analysis of pits formed on Al 2024-T3 in chloride aqueous solution. Appl. Surf. Sci. 2004, 236, 356-365. [CrossRef]

23. Wang, J. The Role of Liquid Film Morphology in Atmospheric Corrosion; Chemical Industry Press: Beijing, China, 2016; pp. 49-56.

24. Zhang, Z. Electrochemical Detection of Atmospheric Corrosion of Aluminum Alloy for Aircraft; Tianjin University: Tianjin, China, 2007; pp. 20-35.

25. Zhang, T.; He, Y.T.; Gao, C. Damage rule of 2A12-T4aluminum alloy with long-term atmospheric corrosion. Acta Aeronaut. Astronaut. Sin. 2015, 36, 661-671.

26. Hao, G.Z.; Xing, L.Y.; Liang, Q.W. Humidity Fixed Point of Saturated Salt Water Solution (2)-Data Source and Salt Solution Selection. Sens. World 1999, 12, 10-14.

27. Sun, S.; Zheng, Q.; Li, D.; Hu, S.; Wen, J. Exfoliation corrosion of extruded 2024-T4 in the coastal environments in China. Corros. Sci. 2011, 53, 2527-2538. [CrossRef]

28. Chen, Q.Z.; Cui, C.J.; Sun, Z.D.; Wang, Y.Y.; Xi, H.Z. Probability Distribution and Variations of Corrosion Damage of LY12CZ Aluminum Alloys. Equip. Environ. Eng. 2005, 3, 1-6.

29. Chen, Y.; Yang, X.; Qin, H. Study on corrosion damage distribution law of aircraft structure. Mater. Sci. Eng. 2002, 20, 378-380.

30. Deng, Z.; Meng-Si, L.I. Dynamic Law of Corrosion Damage Distribution of High Strength Aluminum Alloy Based on Two-parameter Weibull Distribution. Equip. Environ. Eng. 2019, 16, 27-31.

31. Xing, S.B.; Li, X.G.; Li, L. Corrosion Behavior of 7A04 Aluminium Alloy in Xisha Marine Atmosphere. Corros. Prot. 2013, 34, 796.

Publisher's Note: MDPI stays neutral with regard to jurisdictional claims in published maps and institutional affiliations.

(C) 2020 by the authors. Licensee MDPI, Basel, Switzerland. This article is an open access article distributed under the terms and conditions of the Creative Commons Attribution (CC BY) license (http://creativecommons.org/licenses/by/4.0/). 